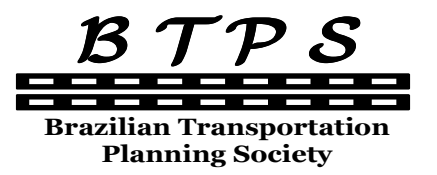

Journal of Transport Literature

Vol. 7, n. 1, pp. 202-211, Jan 2013

Reviews \& Essays
JTL | RELIT

www.transport-literature.org ISSN 2238-1031

\title{
Análise da competitividade do transporte aéreo com base no nível de serviço percebido pelo usuário
}

[Analysis of air transportation competitiveness based on consumer evaluation of service quality ]

\author{
Marcus Vinícius Nascimento* \\ Instituto Tecnológico de Aeronáutica (ITA), Brazil
}

Submitted 30 Apr 2011; received in revised form 29 Nov 2011; accepted 26 Jan 2012

\begin{abstract}
Resumo
Este artigo apresenta uma análise crítica sobre a qualidade percebida pelos usuários de serviços do transporte aéreo. Utilizouse da literatura para determinar como se efetua a avaliação do nível de serviço pelo usuário e como esta pode ser aplicada no sistema de transporte aéreo. Em seguida, foi apresentado um indicativo para mensurar a insatisfação do usuário quanto alguns critérios, como o tempo de espera para a restituição de bagagem, que, segundo especialistas da área, foram responsáveis pelos piores índices de qualidade observada no transporte aéreo de passageiros nos EUA. Os resultados das avaliações apresentadas por este estudo indicam medidas que possam ser tomadas pelas companhias aéreas na mobilização para uma melhor prestação de serviços como a criação de centros de informações para os passageiros e, também, a ação das autoridades reguladoras do serviço do transporte aéreo na aplicação de possíveis multas, visto a observância de problemas com a qualidade do serviço.
\end{abstract}

Palavras-Chave: serviço; usuário; avaliação; companhias; transporte.

\section{Abstract}

This paper presents a critical analysis about the quality service observed by users of air transportation. It uses the specific literature to determine how users evaluate the level of service and how it can be applied on air transportation system. After that, it was submitted an indicative of user poor quality perception against criterions, such as the luggage restitution time, that are considered by specialists, responsible for worst quality perception of air transportation on USA. The results of these evaluation show measures that can be taken by airline companies in the mobilization for a better service as the development of an information center for passengers and also the action of regulatory authorities of air transportation in applying possible penalties when observing issues with service quality.

Key words: service; user; evaluation; companies; transportation.

*Email: mvn.marcus@gmail.com.

\section{Recommended Citation}

Nascimento, M. V. (2013) Análise da competitividade do transporte aéreo com base no nível de serviço percebido pelo usuário. Journal of Transport Literature, vol. 7, n. 1, pp. 202-211.

- JTL/RELIT is a fully electronic, peer-reviewed, open access, international journal focused on emerging transport markets and published by BPTS - Brazilian Transport Planning Society. Website www.transport-literature.org. ISSN 2238-1031.

This paper is downloadable at www.transport-literature.org/open-access. 


\section{Introdução}

O mercado geral de produtos e serviços possui como uma de suas principais características a substitutibilidade, ou seja, a oferta de um amplo leque de opções compatíveis (sejam em questões de disponibilidade, serviço ou preço) aos seus consumidores. As empresas, portanto, necessitam oferecer algum diferencial que as aproximem dos consumidores e que impeça a queda no mercado. Segundo Kolodinsk et al. (1990), muitos autores têm considerado que compreender o nível de serviço esperado pelo consumidor tornou-se, nos últimos anos, uma das questões de diferenciação competitiva das empresas de serviços e um desafio para as companhias aéreas.

Todavia, de acordo com Peters e Waterman (1981, apud Kolodinsk et al. 1990) muitas companhias aéreas ainda não se atentam a este assunto e, consequentemente, não o consideram como fator relevante para ampliação do seu market-share.

Dado este contexto, diversos pesquisadores apontam meios para determinar se os níveis de serviço praticados são avaliados de forma satisfatória pelo consumidor. Um destes meios é a avaliação de reclamações destinadas a certo serviço em um período de tempo. Forbes (2008) colocou que estas queixas, em sua grande maioria, são reflexos de que o serviço recebido pelo consumidor foi avaliado como insatisfatório, ou seja, abaixo de sua expectativa. Outra questão a ser considerada é a necessidade de se determinar quais os critérios exercem mais influência sobre a percepção de qualidade do usuário.

Entretanto, segundo o relatório dos índices de reclamações recebidas nos aeroportos brasileiros apresentado no sítio da ANAC, a quantidade de avaliações recebidas pelas companhias aéreas em relação aos serviços prestados é muito baixa, o que pode ser reflexo de um modelo comportamental dos próprios passageiros que não acreditam em soluções das nuances enfrentadas pelo sistema aéreo através de um ranking de notas.

A base de discussão deste trabalho será o sistema de transporte aéreo. Devido a sua competitividade, alguns autores defendem que o atendimento à satisfação do usuário resulta no aumento da rentabilidade e do desenvolvimento das companhias aéreas (Heskett et al. [1994], apud Bratu e Barnhart [2004]). 
Visto à variedade de serviços oferecidos por este sistema, o presente trabalho será voltado para os mais perceptíveis aos olhos do consumidor e, que, segundo a literatura específica, possuem maior propensão a reclamações contra as companhias aéreas, como os atrasos nos voos e problemas com o manuseio das bagagens.

Desta forma, este estudo tem por objetivo apresentar uma análise crítica sobre a abordagem da satisfação do usuário frente a alguns serviços prestados pelas companhias de transporte aéreo e, que de acordo com pesquisas recentes, têm sido caracterizados pelo excessivo número de reclamações dos passageiros. Busca-se também, identificar possíveis reações das companhias e órgãos regulamentadores do transporte aéreo quanto às variações negativas do nível de serviço. Como exemplo, cita-se a criação de um espaço, na rede mundial de computadores, pelas companhias aéreas em operação no início dos anos 2000, nos Estados Unidos, pelo qual os passageiros eram avisados caso ocorresse algum atraso nos voos e recebiam uma previsão de horário atualizada e a razão pelos atrasos.

Com o intuito de atingir o objetivo proposto, este trabalho foi dividido em três seções. A primeira seção refere-se ao levantamento da literatura necessária para a melhor compreensão de como se dá a avaliação do nível de serviço pelo usuário e da influência que a expectativa do usuário exerce na percepção final de qualidade do serviço. A segunda trata da observação dos resultados obtidos por estudos que avaliam o nível de serviço no sistema de transporte aéreo. A terceira seção baseia-se na apresentação das conclusões obtidas quanto à análise de literatura.

\section{Análise da literatura recente sobre nível de serviço}

Este capítulo refere-se ao embasamento teórico do estudo proposto, o qual será apresentado por meio da seleção de trabalhos científicos considerados relevantes para a discussão do tema. Será apresentada uma pesquisa quanto à forma de avaliação do usuário em relação ao nível de serviço recebido e a análise do nível de serviço aplicada ao sistema de transporte aéreo. 


\subsection{Avaliação do nível de serviço pelo usuário}

De acordo com Peters e Waterman (1981, apud Kolodinsk et al. 1990), a entrega de um serviço que atenda a expectativa do consumidor é imprescindível para a colocação competitiva das empresas.

Bratu e Barnhart (2004) consideram ainda, que, devido a competitividade percebida no mercado do transporte aéreo, o entendimento das expectativas do consumidor pode resultar em altos ganhos financeiros para as companhias aéreas e os participantes do sistema.

Cabe-se, então, do uso de modelos para a avaliação do nível de serviço percebido pelo usuário. Como exemplo, o modelo SERVQUAL (Parasuraman et al. 1985), comumente utilizado em questões de transporte, pressupõe cinco determinantes de qualidade para a prestação de serviços, são eles: confiabilidade, credibilidade, receptividade, segurança e tangibilidade. Por meio destas cinco dimensões propostas pelo modelo, levanta-se quais serão os critérios analisados no problema.

Outros autores completam que a avaliação do nível de serviço pode ser entendida como o "gap", ou intervalo, entre a expectativa do consumidor e a percepção real do serviço, conforme o modelo de Johnston e Clark (2002) ilustrado pela Figura 1.

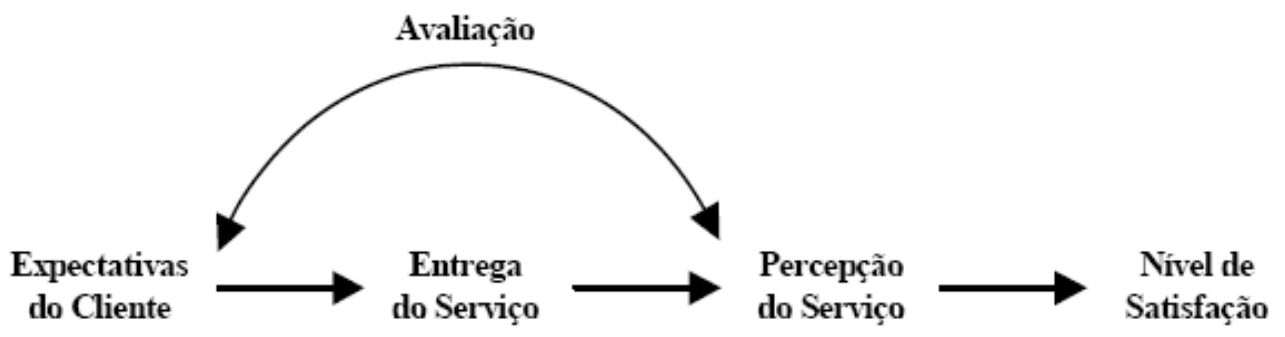

Figura 1 - Modelo de Johnston e Clark (2002) para avaliação do nível de serviço. Fonte: Johnston e Clark, 2002

Por meio da análise do modelo de Johnston e Clark (2002), encerra-se a avaliação do nível de satisfação. 


\subsection{Nível de serviço aplicado ao transporte aéreo}

Além da confiabilidade quanto aos horários de voo e aos cuidados com o manuseio das bagagens, pesquisadores da área propõem outros critérios sensíveis ao usuário no momento da definição de qual companhia aérea utilizar. Bratu e Barnhart (2004) colocam ainda que o efeito preço é um fator de relevância a ser considerado, visto a influência direta deste critério na rentabilidade das empresas do setor.

Outra questão a ser avaliada é que em alguns casos os usuários (ou consumidores) não possuem uma expectativa de qualidade observável ex ante, ou seja, antes do uso ou compra do serviço, mas que se torna observável após o uso (ex post). Neste caso, alguns meios são indicados para se estimar ou até mesmo propor um nível de expectativa ao usuário. Como exemplo de um cenário com expectativa observável ex ante, o site $e$-Bay fornece um sistema de avaliação pública entre as partes compradora e vendedora. Após a finalização de um negócio, ambas as partes podem avaliar o comportamento uma da outra por meio do seguinte sistema: -1 (negativa), 0 (neutra) e 1 (positiva). Observa-se que raramente ocorrem avaliações negativas, algo entorno de $1 \%$ para os fornecedores e $2 \%$ para os consumidores. O resultado deste fato, no entanto, gera um efeito muito maior das pontuações negativas. Resnick et. al (2006) afirmam que, para esta questão, os futuros consumidores criam suas expectativas com base nas avaliações postadas e não na observação direta da qualidade de um fornecedor.

Entretanto, nos casos em que a qualidade se torna observável apenas de forma ex post, a expectativa de qualidade é comumente estimada através de dados e previsões. Após tomar uma previsão para a expectativa de qualidade, pode-se estimar modelos econométricos para determinar a percepção real do serviço. Neste sentido, Forbes (2008) estimou um modelo econométrico - modelo (1) - para a qualidade do serviço do transporte aéreo com base em dados mensais obtidos pelo DOT (Department of Transportation) - Estados Unidos da América, EUA - de voos em atraso e problemas com o manuseio de bagagens, no período de Janeiro de 1988 a Setembro de 2000. Gerou-se, pelo modelo, uma estimativa da expectativa dos usuários para voos pontuais e manuseio das bagagens. A expectativa estimada foi considerada a "expectativa real" em relação a estes critérios. Testou-se a confiabilidade da previsão através da comparação com diversos outros modelos para a expectativa de passageiros. 
Ao tratar a questão da expectativa do usuário, cabe-se uma reavaliação do nível de satisfação obtido através do modelo de Johnston e Clark (2002). Por este modelo é determinado que um alto nível de expectativa pode resultar em índices de satisfação mais baixos ou negativos, visto que a satisfação se dá pela diferença entre a qualidade percebida e expectativa pelo serviço.

No entanto, o nível de insatisfação pode ser previsto através do número de queixas que certo serviço recebe em um determinado período, em função da performance real e prevista. Com base neste argumento, o modelo (1) proposto por Forbes (2008), busca estabelecer uma relação entre o número de reclamações quanto aos atrasos e problemas com o manuseio das bagagens observados e a percepção de serviço real do usuário.

$$
\text { Complaints }_{\mathrm{it}}=\beta_{0}+\beta_{1} \mathrm{~s}_{\mathrm{it}}+\beta_{2} \mathrm{E}\left(\mathrm{s}_{\mathrm{it}}\right)+\varepsilon_{\mathrm{it}}
$$

Neste cenário, o usuário tende a se queixar nos casos em que a qualidade ex post, ou seja, a qualidade percebida após a experiência com o serviço, está abaixo da expectativa de qualidade ex ante.

É importante ressaltar a necessidade do entendimento dos termos do modelo (1): Para $s_{i t}$ e $E\left(s_{i t}\right)$ : performance atual percebida e expectativa de performance no período, respectivamente.

Os parâmetros $\beta_{0}, \beta_{1}$ e $\beta_{2}$ : intercepto, sensibilidade de $s_{\text {it }}$ no número de queixas (Complaints it $_{\text {it }}$ ) - com $\mathrm{E}\left(\mathrm{s}_{\mathrm{it}}\right)$ mantido em constante (ceteris paribus) - e sensibilidade de $\mathrm{E}\left(\mathrm{s}_{\mathrm{it}}\right)$ quanto ao número de queixas - com $\mathrm{s}_{\mathrm{it}}$ e mantido em constante (ceteris paribus) -, respectivamente.

Os termos $i, t$ e $\varepsilon_{i t}$ : companhia aérea, período em meses e "não observáveis" no período $t$ em relação a uma companhia aérea $i$.

Apesar do foco deste trabalho não ser a apresentação e o desenvolvimento de modelos econométricos, vale ressaltar que o modelo (1) foi gerado através de regressão e que os cuidados para se evitar o viés entre os parâmetros foram tomados. O parâmetro previsto para $\mathrm{E}\left(\mathrm{s}_{\mathrm{it}}\right)$ inclui, também, uma previsão de erro quanto a ocorrência de greves e possível falência de alguma companhia, no período em análise. 


\section{Resultados da avaliação}

Entende-se que o modelo (1) de Forbes (2008) resulta no efeito da estimativa da expectativa e da percepção real de qualidade pelo consumidor em relação ao número de queixas para uma companhia aérea $i$ no período $t$.

Diversas inferências podem ser obtidas por este modelo, como exemplo: considerando o efeito ceteris paribus (constante) para a qualidade real, um alto nível de expectativa resulta em maior número de queixas.

Um outro resultante da aplicação do modelo (1) é a redução das queixas conforme a expectativa dos passageiros por atrasos das aeronaves aumenta. Estes resultados são relevantes para as companhias aéreas ao ponto em que a determinação da expectativa do usuário pode exercer grande influência na percepção de qualidade final e, consequentemente, na possibilidade de queixas contra os serviços prestados pelas companhias.

Quanto aos critérios analisados, de acordo com Bratu e Barnhart (2004) dados do DOT revelam que o maior índice de insatisfação e queixas envolvidas com problemas do transporte aéreo nos EUA estão correlacionadas com os cancelamentos e atrasos de voos ou qualquer desvio nos horários programados. De acordo com os dados de avaliação das companhias aéreas publicadas no sítio da ANAC (2009), na internet, o sistema de transporte aéreo brasileiro também possui deficiências quanto a este critério. Observou-se que para os voos domésticos, em cerca de 8000 avaliações no ano, a pesquisa aplicada aos passageiros apresentou como resultados um índice médio pelo quesito pontualidade de 6,79 em um ranking de 0 a 10 (foram avaliadas 14 companhias). No entanto, deve-se atentar para a validade estatística dessa informação em função do baixo número de avaliações em relação ao número de passageiros transportados por ano.

Ainda segundo Bratu e Barnhart (2004) devido os altos índices de atrasos, cancelamento e reagendamento dos voos registrados em 2000, nos Estados Unidos, o conselho público e diversos veículos de mídia buscaram introduzir uma espécie de manual de direto dos passageiros, no qual para cada atraso acima de quinze minutos, cancelamentos e reagendamento dos voos, as companhias aéreas deveriam prover informações sobre a causa 
das interrupções para os passageiros e compensá-los caso houvesse espera superior a duas horas na pista de decolagem ou no desembarque.

Apesar deste manual não ter sido posto em prática, as próprias companhias aéreas se mobilizaram e desenvolveram um espaço no qual fornecem notificações sobre atrasos, cancelamentos e outras informações pertinentes ao passageiro.

\section{Conclusões}

Este estudo apresenta uma análise crítica da literatura relacionada ao serviço observado pelo usuário do sistema de transporte aéreo quanto à competitividade das empresas.

Por meio desta análise, levanta-se a possibilidade de ações que aproximem as companhias aéreas dos consumidores em vias de melhorar a prestação dos serviços aéreos. Para tanto, há a necessidade de se conhecer quais os critérios exercem mais influência sobre os questionamentos dos consumidores. A literatura aponta que, nos Estados Unidos, as questões de pontualidade dos voos e os cuidados com o manuseio das bagagens recebem os maiores índices de reclamações por período. Desta forma, cabe às companhias aéreas determinar formas de conduzir estes questionamentos. Um exemplo disso foi dado quando em um período de problemas com atrasos, reagendamento e cancelamento de voos no ano de 2000, as companhias aéreas que operavam nos EUA desenvolveram um sistema de informações antecipadas ao passageiro, o qual tinha o intuito de minimizar os problemas com as baixas percepções de qualidade no serviço.

Percebe-se também, que a baixa qualidade observada pelo usuário pode resultar na "punição" pública das companhias aéreas quando da revelação de reclamações recebidas. A importância destas publicações se dá a partir do momento em que podem interferir no processo de decisão de novos passageiros por determinada companhia aérea. Todavia, deve-se atentar, nas pesquisas de avaliação, ao tamanho da amostra de passageiros reclamantes. No caso brasileiro, por exemplo, a ANAC apresenta um baixo índice de passageiros reclamantes, o que coloca em dúvida a validade estatística da amostra.

Entretanto, nota-se que as companhias aéreas podem trabalhar em conjunto para a tomada de medidas que aumentem a qualidade percebida pelo passageiro. Cita-se, por exemplo, a criação 
de espaços para informar possíveis atrasos e problemas enfrentados por voos em um dia. Nesta mesma discussão, os órgãos regulamentadores necessitam desenvolver novos meios para observar a prestação do serviço das companhias aéreas e possibilitar a aplicação de possíveis punições para as companhias que apresentarem baixos níveis.

Por fim, esta discussão apresenta um expressivo campo de estudo na relação de avaliar as percepções de serviço do usuário do sistema de transporte aéreo com a competitividade das companhias aéreas. Deixa-se, então, o desafio para as companhias na tomada de medidas que atendam às expectativas dos novos passageiros e a importância do desenvolvimento de novos estudos que abordem outros cenários do sistema de transporte aéreo.

\section{Referências}

Bratu, S., Barnhart, C. (2004) An Analysis of Passenger Delays Using Flight Operations and Passenger Booking Data, Sloan Industry Studies Working Papers.

Forbes, S. J. (2008) The Effect of Service Quality and Expectations on Customer Complaints. The Journal of Industrial Economics, vo. LVI, n. 1, pp. 190-213.

Fraga, R. (2008) Estudo da Interação entre Produtos de Baixa Qualidade vs Produtos de Alta Qualidade no Transporte Aéreo. Journal of Transport Literature, vol. 4, n. 2, pp. 144-159.

Heskett J., Jones T., Loveman G., Sasser E., Schlesinger L. (1994) Putting the Service-Profit Chain to Work, Harvard Business Review, March-April.

Jonhston, R., Clark, G. (2002) Administração das Operações de Serviço. São Paulo: Atlas. 561p.

Kolodinsky, J., Aleong, J. (1990) An Integrated Model of Consumer Complaint Action Applied to Services: A Pilot Study. $C S / D \& C B$, vol. 3, pp. 61-70.

Lovadine, D. (2009) Análise Econométrica Estrutural da Conduta Competitiva: Estudo de Caso do Transporte Aéreo Pós-Liberalização. Journal of Transport Literature, vol. 3, n. 1, pp. 7-39.

Mendes, D. S., Correia, A. R. and Tozi, L. A. (2012) Análise de alternativas para aumento da produtividade e qualidade operacional de terminais de cargas em aeroportos por meio de simulação computacional. Journal of Transport Literature, Vol. 6, n.2.

Parasuraman, A., Berry, L. L., Zeithaml, V. A. (1985) A Conceptual Model of Service Quality and Its Implications for Furure Research, Journal of Marketing, vol. 49, n. 4.

Peters, T., Waterman, R. (1981) In Search of Excellence, (New York: Harper and Row).

Resnick, P., R. Zeckhauser, J. S., Lockwood, K. (2006) The Value Reputation on EBAY: A Controlled Experiment, Experimental Economics, vol. 9, pp. 79-101.

Salgado, L. H., Vassallo, M. D. e Oliveira, A. V. M. (2010) Regulação, Políticas Setoriais, Competitividade e Formação de Preços: Considerações sobre o Transporte Aéreo no Brasil. Journal of Transport Literature, vol. 4, n. 1, pp. 7-48. 
Turolla, F. A., Lima, M. F. F. e Ohira, T. H. (2011) Políticas públicas para a melhoria da competitividade da aviação regional brasileira. Journal of Transport Literature, vol. 5, n. 4, pp. 188-231.

Vassallo, M. D. (2010) Simulação de Fusão com Variações de Qualidade no Produto das Firmas: Aplicação para o Caso do Code-Share Varig-TAM. Journal of Transport Literature, vol. 4, n. 2, pp. 50-100. 